\title{
Fourier analysis of motor current for diagnosis of broken bars in squirrel-cage induction machines
}

\begin{abstract}
Condition monitoring, signal processing and data analysis are the key parts of the induction machine (IM) fault detection scheme. Frequency analysis of the signal acquired through the condition monitoring is commonly applied for motor fault detection. This paper is intended to investigate the ability and effectiveness of Fourier analysis of motor current for broken rotor bar detection. Accordingly, the current signals at different amount of load were measured and analyzed for broken rotor bar diagnosis in a squirrel-cage induction machine.
\end{abstract}

Keyword: Squirrel-cage induction machine; Fault detection; Condition monitoring; Signal processing 